\title{
Superconducting transition temperature and other properties of thin metallic films
}

\author{
Roland E. Allen \\ Department of Physics, Texas A\&M University, College Station, Te; as 77843 \\ (Received 20 March 1975)
}

\begin{abstract}
We point out that the results of (1) Shapoval, for the superconducting transition temperature of a thin film, (2) Nedorezov, for the surface contributions to the density of states, Fermi energy, and specific heat, and (3) Cooper and $\mathrm{Hu}$, for the surface contribution to the density of states, are entirely unphysical because they are obtained from inconsistent models which violate the surface analog of the Friedel sum rule, $\langle\gamma\rangle=\pi / 4$.
\end{abstract}

It has been observed experimentally $y^{1}$ that, for certain weak-coupling superconductors, the transition temperature $T_{c}$ of a thin film is larger than the bulk $T_{c}$. A calculation by Kirzhnits and Maksimov $^{2}$ suggested that surface phonons may be responsible for this enhancement. An experiment involving deposition of noble-gas overlayers on films of various metals, carried out by Naugle $e t$ al. ${ }^{3}$ strongly supported this idea. It was found that the experimental data are in quantitative agreement with a simple model in which the surface phonons have their frequencies increased when the overlayers are deposited.

Shapoval ${ }^{4}$ has suggested a different mechanism involving a boundary condition on the electronic wave functions: He assumes infinite walls at the surfaces of the film. This assumption implies that (a) the value of $\left\langle|\Psi(\vec{r})|^{2}\right\rangle$ increases inside the film, away from the surface, and (b) the Fermi energy also increases. [Here $\left\langle|\Psi(\overrightarrow{\mathrm{r}})|^{2}\right\rangle$ is an average of the square of the electronic wave function.] These are the two effects which, in conjunction, according to Shapoval, produce an increase in $T_{c}$.

In reality, however, both effects are entirely unphysical and result from the use of a model which is not self-consistent in the sense of Hartree or Hartree-Fock theory. In a self-consistent model of a metal, the electrons satisfy what we will call "asymptotic charge neutrality," by which we mean that the sum of the ionic and electronic charge in a region near the surface is equal to zero. As has been shown by Sugiyama, ${ }^{5}$ Langreth, ${ }^{6}$ Appelbaum and Blount, ${ }^{7}$ and Langreth again, ${ }^{8}$ this condition implies a sum rule which is the surface analog of the Friedel sum rule and which we write as

$$
\langle\gamma\rangle=\frac{1}{4} \pi
$$

Here $\langle\gamma\rangle$ is an average phase shift defined in Refs. 5-8. In a semi-infinite free-electron model the electronic wave functions have the form

$$
\begin{aligned}
& \Psi(\vec{r})=\psi(z) \exp \left[i\left(k_{x} x+k_{y} y\right)\right], \\
& \psi(z) \rightarrow \sin \left[k_{z} z-\gamma\left(k_{z}\right)\right], \quad z--\infty
\end{aligned}
$$

if the uniform positive background is in the region $-\infty<z<0$. If an infinite wall is assumed at the surface, $z=0$, then $\psi(0)=0$ and $\gamma\left(k_{z}\right)=0$. Since all the phase shifts equal zero, $\langle\gamma\rangle=0$ and (1) is violated. In order to have a "quasiconsistent" model which at least satisfies the sum rule, we must place the infinite wall just the right distance outside the surface. This argument has been given by Sugiyama. ${ }^{5}$

The condition of asymptotic charge neutrality implies that in the bulk $\left\langle|\Psi(\overrightarrow{\mathrm{r}})|^{2}\right\rangle$ is not changed by the presence of a surface. The electronic wave functions will leak out beyond the surfaces, instead of being pushed back into the interior of the metal from the region near the surfaces by the boundary condition $\psi(0)=0$. They will in fact leak out just enough to insure that the electronic charge density, and consequently $\left\langle|\Psi(\overrightarrow{\mathrm{r}})|^{2}\right\rangle$, are the same in the interior as they would be if there were no surfaces. Also, since the electronic wave functions are not confined to an artificially small volume by the boundary condition $\psi(0)=0$, there will be no increase in the Fermi energy. Appelbaum and Blount ${ }^{7}$ have in fact proved that there is no surface correction to the Fermi energy.

In another paper, Nedorezov ${ }^{9}$ has obtained results for the surface corrections to the electronic density of states, Fermi energy, and specific heat using an infinite-wall model which can incorporate crystallinity. He also obtains a nonzero correction to the Fermi energy, which he suggests can be measured in de Haas-van Alphen experiments. All of Nedorezov's results are unphysical for the reasons given above.

Finally, Cooper and $\mathrm{Hu}^{10}$ have obtained expressions for the electronic density of states with the assumption that either $\psi(0)=0$ or $\psi^{\prime}(0)=0$. According to (3), this implies that either $\langle\gamma\rangle=0$ or $\langle\gamma\rangle=\frac{1}{2} \pi$, so that either assumption violates (1). Thus, the results of Cooper and Hu are unphysical.

In conclusion, we have shown that the results of the three above papers are all unphysical. In particular, both of the effects invoked by Shapoval to explain the change in $T_{c}$ for a thin film result from the use of an incorrect model. 
${ }^{1}$ W. Buckel and R. Hilsch, Z. Phys. 131,420 (1952); 138, 109 (1954).

${ }^{2}$ D. A. Kirzhnits and E. G. Maksimov, Zh. Eksp. Teor。 Fiz. Pis'ma Red. 2, 442 (1965) [JETP Lett. 2, 274 (1965) ]. Shapoval (Ref. 4) says that the assumed increase in the electron-electron interaction near the surface is associated with the presence of surface modes (Rayleigh waves).

${ }^{3}$ D. G。 Naugle, J. W. Baker, and R. E. Allen, Phys. Rev. B 7, 3028 (1973).

${ }^{4}$ E. A. Shapoval, Zh. Eksp. Teor. Fiz。Pis'ma Red. $\underline{5}$, 57 (1967) [JETP Lett. 5, 45 (1967)].

${ }^{5}$ A. Sugiyama, J. Phys. Soc. Jpn. 15, 965 (1960)。

${ }^{6}$ D. C. Langreth, Phys. Rev. B $\underline{5}, 2842$ (1972).

${ }^{7} \mathrm{~J}$. A. Appelbaum and E. I. Blount, Phys. Rev. B 8,483 (1973). We are not including the complications due to crystallinity, possible surface states, etc., because they are irrelevant here.

${ }^{8}$ D. C. Langreth, Phys. Rev. B 11,2155 (1975).
${ }^{9}$ S. S. Nedorezov, Zh. Eksp. Teor. Fiz. 51, 868 (1966) [Sov. Phys. -JETP 24, 578 (1967)].

${ }^{10} \mathrm{~L}$. N. Cooper and S. Hu, in Electronic Density of States, edited by L. H. Bennett, (NBS Special Publication No. 323, (U. S. GPO, Washington, DC, 1971), p. 473。 We mention that the results for the surface density of states obtained in this paper are special cases of the general result obtained by V. E. Kenner and R. E. Allen [Phys. Rev. B $11,2858(1975)$ ], when $\gamma\left(k_{F}\right)=0$ or $\gamma\left(k_{F}\right)=\frac{1}{2} \pi$. However, since $\langle\gamma\rangle=\frac{1}{4} \pi$ and $\gamma\left(k_{k}\right)$ should increase with $k_{z}$, as it does in the results displayed in Ref. 5 , we should have $\gamma\left(k_{F}\right)>\frac{1}{4} \pi_{\text {。 }}$ The assumption $\gamma\left(k_{F}\right)=0$ gives, for example, the wrong sign for the surface specific heat, which contains a factor $\left[\gamma\left(k_{F}\right)-\frac{1}{4} \pi\right]$ according to Kenner and Allen. Cooper and Hu state, "One might consider a metal-vacuum boundary to simulate $\psi=0$ at (the) boundary," which would imply $\gamma=0$ for a metal-vacuum interface. 